\title{
Fault condition recognition of rolling bearing in bridge crane based on PSO-KPCA
}

\author{
He Yan ${ }^{1, a}$ and Wang Zongyan ${ }^{1}$ \\ ${ }^{1}$ School of Mechanical and Power Engineering, North University of China, Taiyuan, 030051 , China
}

\begin{abstract}
When the rolling bearing in bridge crane gets out of order and often accompanies with occurrence of nonlinear behaviours, its fault information is weak and it is difficult to extract fault features and to distinguish diverse failure modes. Kernel principal component analysis (KPCA) may realize nonlinear mapping to solve nonlinear problems. In the paper the particle swarm optimization (PSO) is applied to optimization of kernel function parameter to reduce its bind set-up. The optimal mathematical model of kernel parameters is constructed by means of thought of fisher discriminate functions .And then it is used to bridge crane rolling bearing simulated faults recognition. The simulation results show that KPCA optimized by PSO can effectively classify fault conditions of rolling bearing.It can be concluded that nonlinear mapping capability of KPCA after its function parameter by PSO is greatly improved and the KPCA-PSO is very suit for slight and incipient mechanical fault condition recognition.
\end{abstract}

\section{Introduction}

Rolling bearing is very important component of drive system in bridge crane. When it gets out of order, its fault information is weak and it often accompanies with occurrence of nonlinear behaviours, so it is difficult to extract fault features and to distinguish diverse failure modes, and it is disadvantage of the recognition and diagnosis of fault condition. Kernel principal component (KPCA) analysis may realize nonlinear mapping via kernel function [1]. It maps the input vector into a high dimensional feature space by use of the nonlinear mapping, aimed to remain good divisibility, then get nonlinear principal component of initial data by PCA of mapping data in a high dimensional space. KPCA is more applicable to solve nonlinear problems [2]. However, to given data sets, the classification of KPCA is influenced by itself parameter of kernel function, consequently, it is of utmost importance to choose optimum kernel function parameter (namely Kernel parameter). Currently, it mainly relies on a great deal number of experiments for sure or uses method of crossing tests, which is not only timeconsuming and low efficiency, but also fails to make sure the kernel function is in optimal parameters. Therefore, the study of kernel function parameters optimization method for fault classifier and its performance optimization has the vital significance [3].

Particle swarm optimization (PSO) is a kind of global optimization method of parallel based on swarm intelligence, and it has been widely applied in function optimization [4]. The paper tries to solve the optimization problem of kernel parameter by the combination of Fisher discriminate function and PSO algorithm. Firstly, parameter optimization model of kernel function is constructed,

\footnotetext{
${ }^{a}$ Corresponding author : He Yan@zbhy91@163.com
} 
then the global optimal solution for it is searched by PSO, and optimized KPCA will be applied bridge cane rolling bearing fault condition recognition. At last the recognition efficiency by PSOKPCA will be analyzed.

\section{Particle Swarm Optimization algorithms}

In the PSO algorithm, each particle moves according to its velocity in the search space. The whole optimization process is that particle adjusting to its position and speed based on its optimal location and that of other individuals in its neighbour, then to accelerate moving toward global optimal value. The standard particle swarm algorithm is shown in equation (1) (2).

$$
\begin{gathered}
v_{i d}(t+1)=\omega v_{i d}(t)+c_{1} r_{1}\left(p_{i d}-x_{i d}(t)\right)+c_{2} r_{2}\left(p_{g d}-x_{i d}(t)\right) \\
x_{i d}(t+1)=x_{i d}(t)+v_{i d}(t+1)
\end{gathered}
$$

Where $x_{i d}(t)$ is the current position of the particle $i, p_{i d}$ is superior position of particle $i$, which searches until now, $p_{g d}$ is the entire particle swarm' s superior position. $i=1,2, \ldots, \mathrm{m}, d=1,2, \ldots, D$; $c_{l}$ and $c_{2}$ are two positive constants, called cognitive learning rate; $r_{1}$ and $r_{2}$ are random numbers in range $[01] ; v_{i d} \in\left[-v_{\max }, v_{\max }\right] ; v_{\max }$ is constant. The selected condition of stopping iteration is the max iterative times $T_{\max }$ or the best position of particle searched till now meets for thresholds $\varepsilon$; $\omega$ is positive constants, called inertia factor which is linearly reducing with iteration $t$ according to equation (3).

$$
\omega=\omega_{\max }-\frac{\omega_{\max }-\omega_{\min }}{T_{\max }} t
$$

Where $\omega_{\max }, \omega_{\min }$ are the maximum and the minimum inertia weight, typically $\omega_{\max }=1.2$, $\omega_{\min }=0.4$.

\section{The kernel parameter optimization based on PSO}

\subsection{The Principle of Kernel principal component analysis}

Kernel principal component analysis (KPCA) will project input space into feature space through nonlinear mapping, and then make a principal component analysis on mapping data in feature space, finally obtain the projection of the data in nonlinear principal component. The nonlinear mapping is realized by inner product operation, which is only required kernel function calculating for inner product in the original space, and without concerning specific form of nonlinear mapping, so called as KPCA. By choosing different forms of kernel function, it can deal with a great deal of nonlinear problems [5]. For kernel method, most current kernel function which is researched mainly contain polynomial kernel function, RBF kernel function and neural network kernel function and so on, however, to choose different kernel functions can got very similar classification results [1,4].To simplify quantities of calculation, only RBF kernel function is chosen here, the specific form of kernel function is as equation (4).

$$
k(x, y)=\exp \left(-\frac{\|x-y\|^{2}}{\sigma}\right)
$$




\subsection{The optimization model of kernel parameter is constructed}

The main idea of KPCA transformation is to map training sample data into a high dimensional feature space, then to establish an optimal separating hyperplane for the distance between two kinds of data points and the hyperplane being the largest. However, in traditional pattern recognition, the idea of Fisher discriminate function is just opposite. Its basic thought is to find an optimal projection direction, along which original data is projected into a straight line in order to distinguish better data after projection; the distance between different data modes is as big as possible whereas the data discrete degree between the same patterns is as small as possible. Therefore an optimization method of kernel parameters can be achieved by thought of Fisher discriminate functions [6].Kernel parametric model optimized by PSO based on fisher norm is established as follows.

Assumption that parameters $\mathrm{X}_{1}\left(x_{11}, x_{12}, \ldots, x_{1 i}\right)$ and $\mathrm{X}_{2}\left(x_{21}, x_{22}, \ldots, x_{2 \mathrm{j}}\right)$ are two kinds of characteristic samples in a feature space, among which $i=1,2, \ldots, n_{1}, j=1,2, \ldots, n_{2}$. The mean vectors for the two kinds of samples in feature space are respectively as equation (4)(5).

$$
\begin{aligned}
& t_{1}=\frac{1}{n_{1}} \sum_{i=1}^{n_{1}} \Phi\left(x_{1 i}\right) \\
& t_{2}=\frac{1}{n_{2}} \sum_{\mathrm{j}=1}^{n_{2}} \Phi\left(x_{2 \mathrm{j}}\right)
\end{aligned}
$$

The square of the distances between classes is as equation (6).

$$
\begin{aligned}
& \mathrm{d}_{\mathrm{b}}=\left\|\mathrm{t}_{1}-\mathrm{t}_{2}\right\|=\left(\mathrm{t}_{1}-\mathrm{t}_{2}\right)^{\mathrm{T}}\left(\mathrm{t}_{1}-\mathrm{t}_{2}\right) \\
& =\frac{1}{n^{2}} \sum_{i=1}^{n_{1}} \sum_{j=1}^{n_{2}} k\left(x_{1 i}, x_{1 j}\right)-\frac{2}{n_{1} n_{2}} \sum_{i=1}^{n_{1}} \sum_{j=1}^{n_{2}} k\left(x_{1 i}, x_{2 j}\right)+\frac{1}{n_{2}^{2}} \sum_{i=1}^{n_{1}} \sum_{j=1}^{n_{2}} k\left(x_{2 i}, x_{2 j}\right)
\end{aligned}
$$

The square of inner-class discrete degree is as equation (7)(8).

$$
\begin{aligned}
& \mathrm{d}_{w 1}=\frac{1}{n_{1}} \sum_{i=1}^{n_{1}}\left\|\Phi\left(x_{1 i}\right)-\mathrm{t}_{1}\right\|^{2}=\sum_{i=1}^{n_{1}} k\left(x_{1 i}, x_{1 i}\right)-\frac{1}{n_{1}} \sum_{i=1}^{n_{1}} \sum_{j=1}^{n_{2}} k\left(x_{1 i}, x_{1 j}\right) \\
& \mathrm{d}_{w 2}=\frac{1}{n_{1}} \sum_{\mathrm{j}=1}^{n_{2}}\left\|\Phi\left(x_{2 i}\right)-\mathrm{t}_{2}\right\|=\sum_{j=1}^{n_{2}} k\left(x_{2 j}, x_{2 j}\right)-\frac{1}{n_{2}} \sum_{i=1}^{n_{1}} \sum_{j=1}^{n_{2}} k\left(x_{2 i}, x_{2 j}\right)
\end{aligned}
$$

According to fisher discriminate criterion, fitness function of PSO is set up as equation (9).

$$
\text { fitness }=f(\sigma)=d_{w 1}+d_{w 12} / d_{b}
$$

Seeking for the minimum value of fitness function $f(\sigma)$ can ensure that the between-class scatter is maximal; at the time the parameter $\sigma$ can be $\sigma^{*}$ on the condition that $f(\sigma)$ changes to be steady at the outset.

\subsection{Steps of kernel parameters optimized by PSO}


The step of the kernel function parameter optimized by PSO is as follows:

1) Through equation (7), (8), (9), the square value of distance between classes and that of within-class scatter are calculated, respectively;

2) Fisher discriminate function is constructed by the formula (10), and regarded as a fitness for PSO;

3) Some conditions are given such as value range of the parameters $\omega_{\max }, \omega_{\min }, m, T_{\max }, c_{1}$ and $c_{2}, V_{\max }$;

4) Initial population $\sigma$ is randomly generated; similarly, the value of individual fitness and the global fitness value of population are calculated;

5) Whether iterative step meets the termination condition is determined. If $t<T_{\max }$, the step (4) and (5) will be repeated; otherwise, the current solution will be output as the optimal solution, and the algorithm is terminated.

\section{Rolling bearing of bridge cane fault recognition based on PSO-KPCA}

\subsection{Construction of bearing feature parameter set}

Faults simulation experiment were done through the rolling bearing of bridge crane researched as object in laboratory, for example normal condition, damage of bear cage, scratch of bear inner race, and flaw of bear outer ring. Figure1shows test system of simulated faults experiment, and Figure 2 shows two typical failure forms of rolling bearing .Based on measuring the acceleration signal from different rotational speeds of six measuring points in the gearbox cover, some operations are done such as signal processing, extraction of characteristic parameters for time domain and frequency domain as the original feature parameters set. 60 groups of different faults samples (30 groups of training samples and 30 groups of test samples) extracted from features sets are applied to KPCA and identification of bearing faults condition [7].

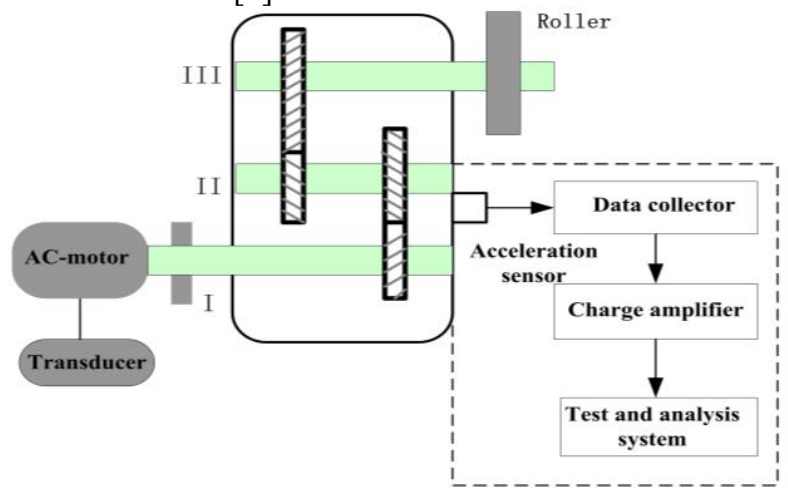

Figure 1. Test system diagram of simulated fault diagnosis experiment of rolling bearing

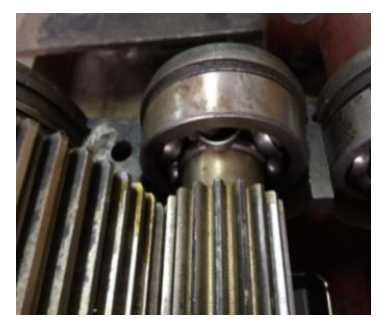

(a) Damage of bear cage

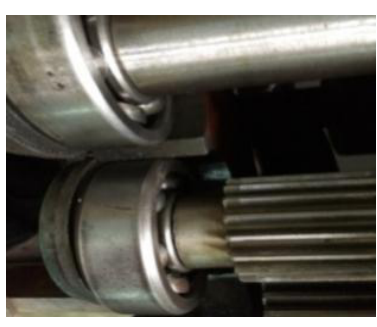

( b) Flaw of bear outer ring

Figure 2 Typical failure forms of rolling bear in bridge cane 


\subsection{Kernel function parametric optimization implemented by PSO}

In KPCA, the width of the radial basis kernel function $\sigma$ can ensure a good performance of kernel function. For training samples, PSO is adopted to optimize kernel parameters, among which parameters are set as $m=20, T_{\max }=50, V_{\max }=1, \omega_{\max }=1.2, \omega_{\min }=0.4, c_{1}=c_{2}=2$. The optimization width values $\sigma^{*}$ of radial basis function between condition model of rolling bearing are obtained, which are shown in table 1. The optimal values based PSO are the basis for parameters setting of bearing fault feature sets by KPCA.

Table1. Kernel parameter optimization results of different condition samples of bearing

\begin{tabular}{|c|c|}
\hline condition model of rolling bearing & $\sigma^{*}$ \\
\hline Normal condition and scratch of bear inner race & 10.24 \\
\hline Damage of bear cage and flaw of bear outer ring & 0.81 \\
\hline Damage of bear cage and scratch of bear inner race & 17.48 \\
\hline Scratch of bear inner race and flaw of bear outer ring & 3.22 \\
\hline
\end{tabular}

\subsection{Fault condition recognition of based on PSO- KPCA}

In the research, 30 original feature sets on normal condition are trained, and tests are done through 30 different groups of data features such as damage of bear cage and the failures of bearings inner race and outer ring; Figure 3 shows the projection from the first principal component to the second principal component, extracted by KPCA. Among which, non-linear principal component for KPCA are unified dimension parameters.

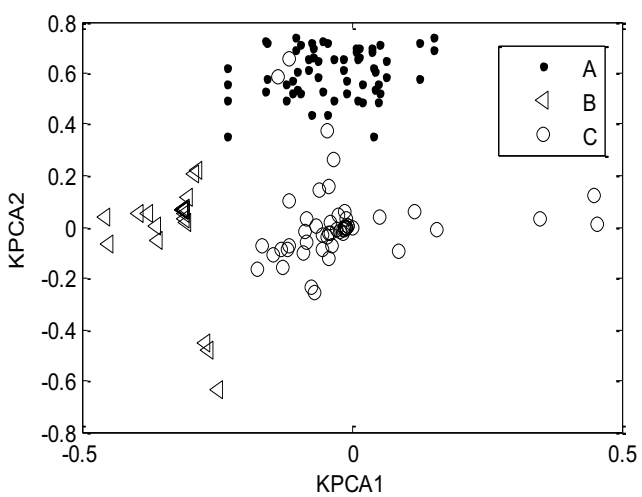

(a)

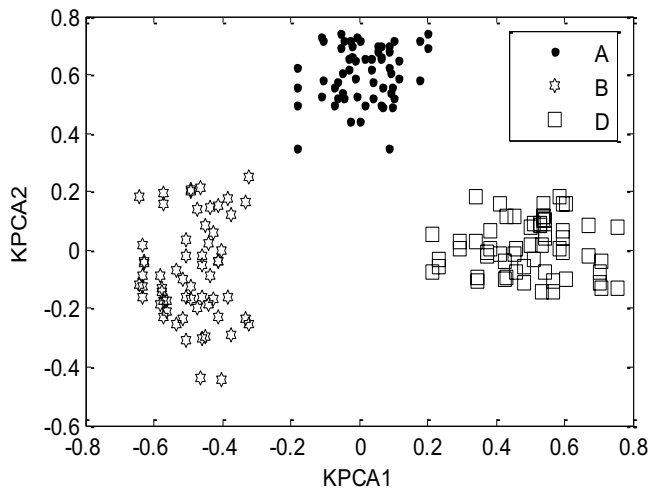

(b)

A: Normal condition, B: Damage of bear cage, C: Scratch of bear inner race, D: Flaw of bear outer ring Figure 3. The first and second principal component projection KPCA of different conditions of bearing

As can be seen from Figure 3 (a)(b), KPCA-PSO analysis can clearly distinguish the bearing fault types. The principal component characteristics of normal condition data are self-assortment, and those of damage of bear cage and scratch of bear inner race characteristics of the are relatively scattered, but away from the normal centre of clustering, clearly belong to different categories. Additionally, the inner-class distance of KPCA is small, whereas the distance between classes of KPCA is larger. So, KPCA optimized by PSO shows a greater advantage in the non-linear analysis, and can accurately identify the fault condition of rolling bearing. 


\section{Conclusions}

PSO algorithm is used to optimize the parameters of kernel function for reduction the blindness of KPCA in parameters set and applied into bearing fault conditions recognition to improve the performance of KPCA. It can effectively identify bearing fault modes such as normal condition, damage of bear cage, scratch of bear inner race, and flaw of bear outer ring. The effect of failure identification is obviously superior. Research results show that kernel principal component analysis based on PSO has advantages in the nonlinear feature extraction of mechanical fault and is very suit for light and incipient mechanical fault condition recognition.

\section{References}

1. Sh .Renping, H. Wentao, W. Yayun, Q. Xiankun .The fault feature extraction and classification of gear using principal component analysis and kernel principal component analysis based on the wavelet packet transform. IMEKO.54 (2014) 118 - 132

2. H. Qingbo, K. Fanrong, Y. Ruanqiang. Subspaced-based gearbox monitoring by kernel principal component analysis . MSSP, 21 ( 2007) 1755-1772

3. X. Yingqun Xiao,F. Lianggui. A novel neural-network approach of analog fault diagnosis based on kernel discriminate analysis and particle swarm optimization. WFSC , 12 (2012) $904-920$

4. B. Scholkopf, A. Smola, K.R. Muller.Nonlinear principal component analysis as a kernel eigenvalue problem. $N C, \mathbf{1 0}$ (1998)1299-1319

5. H. Xuewen. Study on theory and method of fault intelligent diagnosis based on support vector machine [D]. South University Of China, Changsha, 12 (2004).

6. Zh.Ying, Z. Hongfu, B.Fang. Classification of fault location and performance degradation of a roller bearing. IMEKO, 46 (2013) 1178 - 1189

7. W. Xinfeng, Q. Jing, L. Guangjun. Kernel Function Optimization in Kernel Principle Component Analysis and Its Application to Feature extraction of gear faults. Journal of Vibration,Measurement \&Diagnosis,27,1( 2007) 62-64 